\title{
Citation Classics on Dental Caries: A Systematic Review
}

\author{
Anas Imran Arshad 1,2,- $\quad$ Paras Ahmad ${ }^{3} \quad$ Paul M.H. Dummer ${ }^{4} \quad$ Mohammad Khursheed Alam $^{5}$ \\ Jawaad Ahmed Asif ${ }^{6} \quad$ Zuliani Mahmood ${ }^{1} \quad$ Normastura Abd Rahman ${ }^{7} \quad$ Noraida Mamat ${ }^{1}$
}

1 Paediatric Dentistry Unit, School of Dental Sciences, Universiti

Sains Malaysia, Kota Bharu, Kelantan, Malaysia

2Paedodontics Department, Rashid Latif Dental College, Rashid Latif

Address for correspondence Zuliani Mahmood, DClinDent, Paediatric Dentistry Unit, School of Dental Sciences, Universiti Sains Malaysia,

Medical College, Lahore, Pakistan

${ }^{3}$ Department of Oral Medicine, School of Dental Sciences, Universiti

Sains Malaysia, Kota Bharu, Kelantan, Malaysia

${ }^{4}$ School of Dentistry, College of Biomedical and Life Sciences,

Cardiff University, Cardiff, United Kingdom

${ }^{5}$ Orthodontic Department, College of Dentistry, Jouf University,

Sakaka, Kingdom of Saudi Arabia

${ }^{6}$ Department of Oral and Maxillofacial Surgery, School of Dental

Sciences, Universiti Sains Malaysia, Kota Bharu, Kelantan, Malaysia

${ }^{7}$ Department of Dental Public Health, School of Dental Sciences,

Universiti Sains Malaysia, Kota Bharu, Kelantan, Malaysia

\begin{abstract}
Keywords

- citation classics

- dental caries

- systematic review

- citation analysis

- Streptococcus mutans

- bibliometrics

Objective A systematic search was performed for the identification and analysis of the 100 most often cited articles on dental caries and to highlight the changing trends in the field of dentistry over time.

Materials and Methods The search was performed without any restriction on the study design, publication year, or language using the Web of Science (WoS) group of Clarivate Analytics enabling the search through "All Databases." Based on the citation count as available in WoS, the articles were sorted in a descending manner. Information regarding each article was then extracted, which included its authorship, counts of citation (in other databases), citation density, current citation index (2019), publication year, country of publication, journal of article, evidence level based on study design, and keywords description.

Results The count of citation for each article varied in each database, that is, 175 to 2,003 in WoS, 89 to 1,981 in Scopus, and 126 to 3,492 when searched in Google Scholar. The highest number of articles $(n=10)$ related to dental caries were published in 2004. A total of 301 authors made valuable contributions to this field, out of which J.D. Featherstone had coauthored 6 articles. A significant negative correlation $(p<0.01)$ was found between the age of the article and the citation density $(r=-0.545)$. However, a nonsignificant correlation ( $p=0.952$ ) occurred between the age of publication and the citation count $(r=0.006)$.

Conclusion The results of this systematic review provide a critical appraisal of the context underpinning scientific developments in the field of dental caries and also highlighted trends in clinical management and research.
\end{abstract}

\section{Introduction}

"Dental caries is a biofilm-mediated, sugar-driven, multifactorial, dynamic disease that results in the phasic demineralization and remineralization of dental hard tissues." ${ }^{1}$
A multifactorial origin has been identified in which the presence of acidogenic bacteria, salivary disturbances, and sugar consumption/frequency are known to play a vital role in disease progression. ${ }^{2}$ Caries is a common chronic disease which has a high prevalence rate among adults and 
children. ${ }^{3}$ Approximately 2.4 billion people with untreated lesions have been estimated worldwide. ${ }^{4}$ Untreated carious lesions commonly lead to functional, aesthetic, and psychological problems, as well as a poor quality of life..$^{5}$ Delay in the treatment of a carious tooth eventually leads to pulpal involvement and painful mastication potentially resulting in indigestion, ${ }^{6}$ malnutrition, ${ }^{7}$ and systemic infections, which in turn increase the treatment need and consequently increase the cost of dental and medical care provided to the patient.

The citation count of an article is an indicator of its impact in its respective field. A classic article is defined as an article having secured a citation count of 100 or above. ${ }^{8}$ The growth of a particular field or specialty can be studied by performing a bibliometric analysis. It also provides vital information regarding the prominent areas of individual medical and dental specialties. ${ }^{9}$ Various bibliometric analyses have documented the citation classics in the field of dentistry, ${ }^{10-20}$ but no study has been performed to study the characteristic features of the publications on caries research which have been cited most often.

The current study is focused on identification and analysis of the top 100 publications which have been cited most often regarding dental caries and to highlight the change in current trends, centers of excellence in caries research, dominant types of methodology, and technological developments made during the elapsed time.

\section{Materials and Methods}

\section{Protocol}

The Preferred Reporting Items for Systematic Reviews and Meta-Analyses (PRISMA) guidelines were followed for the preparation of this report. ${ }^{21}$

\section{Search Strategy}

The Web of Science (WoS) group of Clarivate Analytics was used to perform a literature search on April 1, 2019 enabling the search through "All Databases." The title section was searched using the search terms described below in inclusion criteria. The database was searched without any restriction on the language of the article, study design, or publication year. Based on the WoS results, 31,584 articles were retrieved which were then arranged according to number of citations. One hundred articles with the largest number of citations were tabulated. A manual cross-matching of citation count was performed on the Elsevier's database (Scopus) and the Google Scholar (GS). The top 100 "classic" publications were selected by two reviewers independently and the final list was unanimously agreed upon. The - Supplementary Fig. $\mathbf{S 1}$ (online only) shows the process of selection of articles according to PRISMA guidelines.

\section{Inclusion Criteria}

Inclusion criteria were the presence of "caries" OR "carious" OR "cariogenic" OR "cariology" OR "tooth decay" OR "teeth decay" OR "tooth cavity" OR "teeth cavity" OR "tooth cavities" OR "teeth cavities" OR "dental cavity" OR "dental cavities" OR "dental decay" in the title of the article and publication in a peer-reviewed journal.

\section{Exclusion criteria}

Articles having less than 100 citations according the WoS "All Databases" and publications in journals with low $(\leq 0.500)$ or no impact factor were excluded.

\section{Data Extraction}

Article authorship, title of article, counts of citation (WoS, Scopus, and GS), publication year, citation density, current citation index (CCI) (2019), institution and country of publication, journal of article, evidence level based on methodology, and keywords description were noted for each selected publication.

\section{Graphical Analysis}

VOSviewer is a free software platform which is used for bibliometric mapping based on visualization of similarities. ${ }^{16}$ We used this software to visualize the clusters of author keywords which have been used in the top-cited articles. This method of mapping has been used previously and provides a simplistic representation of data. ${ }^{18}$

\section{Statistical Analysis}

IBM SPSS Statistics processes version 24 for Windows was used to perform descriptive and bivariate analyses. The Shapiro-Wilk test was performed to check data normality. Based on distribution and normality of the data, mean (standard deviation) or median (interquartile range) were calculated. The Kruskal-Wallis test was performed to analyze the median differences between the independent groups and differences within each group was checked by post hoc testing. The Mann-Kendall trend test was performed to analyze an increase or decrease in the time-dependent trends. To evaluate the correlation between the age of the journal and the publication count in the journal the Spearman-rank test was performed. A value of $p<0.05$ was considered statistically significant.

\section{Results}

\section{Authorship}

Three hundred and one authors made contributions to the list of top 100 "classics." J.D. Featherstone $(n=6)$ had the highest publication count followed by P. Axelsson $(n=5)$, J. Lindhe $(n=5)$, B. Nyvad $(n=4)$, N.B. Pitts $(n=4)$, B. Krasse $(n=3)$, and J. van Houte $(n=3)$. Twenty-two authors contributed to two "classics" each, among the list of top 100 "classics." The -Supplementary Table S1 (online only) shows the number of instances at which an author appeared as the first author, as the corresponding author, as the first and the corresponding author, and as the coauthor.

\section{Citation Count, Citation Density, and Current Citation Index}

A total of 297,496 (WoS), 27,713 (Scopus), and 53,648 (GS) citations were calculated for the list of top 100 "classics." 
The range of citations varied from 175 to 2,003 (WoS), 89 to 1,981 (Scopus), and 126 to 3,492 (GS). The average number of annual citations is termed as citation density which was calculated as 297.49 (WoS), 277.13 (Scopus), and 536.48 (GS) collectively. "Role of Streptococcus mutans in human dental decay" (citation density $=62.59$ ) was the most cited "classic" article with 2,003 (WoS), 1,981 (Scopus), and 3,492 (GS) citations. ${ }^{22}$ "Genome sequence of Streptococcus mutans UA159, a cariogenic dental pathogen" (citation density $=69.68$ ) was ranked as the second "classic" article with 1,115 (WoS), 652 (Scopus), and 955 (GS) citations. ${ }^{23}$ "Dental caries" (citation density $=82.27$ ) was ranked as the third "classic" article with 905 (WoS), 952 (Scopus), and 1,954 (GS) citations. ${ }^{24}$ "Global burden of untreated caries: A systematic review and meta-regression" had the highest citation density of 89.0.25 The list of top 100 "classics" on dental caries are presented in -Table $\mathbf{1}$ along with their citation counts in WoS, Scopus, and GS and their citation density.

The Shapiro-Wilk test revealed that the data distribution of citation count, age of publication, and citation density were not normal $(p<0.01)$. The trend towards a greater number of citation counts with publication age was not significant ( $r=0.006, p=0.952$ ) as shown in - Fig. 1. However, a negative trend towards an increased citation density with increasing time since publication was found to be significant $(r=-0.545, p<0.01)$ as shown in - Fig. 2.

According to the CCI based on the year 2019, two out of the top six articles were systematic reviews, three were field expert reviews, and one was caries management system/ tool. These findings highlight the sustainability and current relevance of information provided in systematic reviews and expert opinions.

\section{Publication Year}

The "classic" publications on the topic of caries were published between $1954^{26}$ and $2015^{25}$ as displayed in - Fig. 3 . Ten articles were published in 2004 which was the highest number of publications in any one year. Chronologically, 2 publications in 1950s, 10 in 1960s, 12 in 1970s, 10 in 1980s, 18 in 1990s, 44 in 2000s, and 4 since 2010 were classified as "classics." From 2000 to 2005, peaks were noticed in the number of "classic" articles, that is, 32 . Four out of 100 "classics" were published after the year 1999.

\section{Institution and Country of Publication}

The corresponding author of each article and their affiliations revealed that authors from 13 countries made contributions toward caries research. The highest number of publications originated from the United States $(n=45)$ followed by Sweden ( $n=14)$, United Kingdom ( $n=11)$, Switzerland $(n=7)$, Denmark $(n=5)$, Japan $(n=4)$, Finland $(n=4)$, Netherlands $(n=3)$, Norway $(n=2)$, Brazil $(n=2)$, Germany $(n=2)$, France $(n=1)$, and Australia $(n=1)$.

The highest number of publications originated from the "University of Gothenburg, Gothenburg, Sweden" $(n=7)$ among 50 other institutions, "School of Dentistry, University of Michigan, Ann Arbor, Michigan, United States"
( $n=6)$, "National Institutes of Health, Bethesda, Maryland, United States" $(n=5)$, "School of Dentistry, University of California at San Francisco, San Francisco, California, United States" ( $n=5)$, "Forsyth Dental Center, The Forsyth Institute, Massachusetts, United States" $(n=4)$, "Royal Dental College, Aarhus University, Aarhus, Denmark" $(n=4)$, "School of Dentistry, University of Lund, Lund, Sweden" ( $n=3)$, "Dental Institute, King's College London, London, United Kingdom" ( $n=3)$, "School of Dentistry, Karolinska Institutet, Solna, Sweden" $(n=3)$, and "Institute of Dentistry, University of Helsinki, Helsinki, Finland" $(n=3)$.

\section{Journal of Publication}

The 100 "classic" publications on the topic of caries were published across 40 peer-reviewed journals. The journals associated with the most number of publications were "Journal of Dental Research" ( $n=19)$, "Caries Research" $(n=17)$, and "Archives of Oral Biology" $(n=6)$. The impact factors of journals ranged from 0.784 (Pan American Journal of Public Health) to 53.254 (The Lancet). - Table 2 summarizes the complete list of all journals.

A significant trend $(p<0.05)$ occurred between a specific journal age and the quantity of "classics" published in that journal $(r=0.321)$. However, a statistically nonsignificant trend ( $p=0.196)$ occurred between the quantity of "classics" published in a specific journal and the impact factor of that journal.

\section{Methodological Design}

The most common study design among "classics" was review-type $(n=40)$, clinical studies $(n=23)$, laboratory studies $(n=16)$, animal studies $(n=15)$, new classification/ tool/technique $(n=5)$, and cohort studies $(n=1)$. Statistical significance was not detected $(p=0.808)$ when exploring the median difference in the citation count per "classic," between review-type articles 238 (range: 175-2,003), clinical studies 258 (range: 176-567), laboratory studies 224 (range: 184-403), animal studies 250 (range: 176-442), and new classification/tool/technique 207 (range: 174-486).

\section{Evidence Level}

The top 100 most-cited "classic" articles could be categorized into all evidence levels. The greatest number of articles were within evidence level $\mathrm{V}(N=41)$ followed by evidence level IV ( $n=26)$, evidence level III $(n=16)$, evidence level II $(n=13)$, and evidence level $\mathrm{I}(n=4)$. Among these evidence levels, the citation density $(r=0.088, p=0.383)$ and the total citation counts $(r=-0.178, p=0.077)$ did not vary significantly.

\section{Keywords}

A total of 190 unique keywords were found among these "classic" articles. The frequency of occurrence of these keywords were counted as dental caries $(n=24)$ followed by caries $(n=16)$, Streptococcus mutans $(n=14)$, fluoride $(n=6)$, dentin $(n=5)$, lactobacillus $(n=5)$, saliva $(n=5)$, Actinomyces $(n=4)$, dental plaque $(n=4)$, and gingivitis $(n=4)$. - Fig. 4 shows the network analysis of the keywords. 
Table 1 Ranking list of the top 100 most cited articles on caries research

\begin{tabular}{|c|c|c|c|c|c|c|}
\hline Rank & Title of the article & $\begin{array}{l}\text { No. of } \\
\text { citation } \\
\text { (Web of } \\
\text { Science) }\end{array}$ & $\begin{array}{l}\text { No. of } \\
\text { citation } \\
\text { (Scopus) }\end{array}$ & $\begin{array}{l}\text { No. of } \\
\text { citation } \\
\text { (Google } \\
\text { Scholar) }\end{array}$ & $\begin{array}{l}\text { Citation } \\
\text { density }\end{array}$ & CCI 2019 \\
\hline 1 & $\begin{array}{l}\text { Loesche WJ: Role of Streptococcus mutans } \\
\text { in human dental decay. Microbiol Rev } \\
\text { 1986;50:353-380 }\end{array}$ & 2,003 & 1,981 & 3,492 & 62.59 & 64 \\
\hline 2 & $\begin{array}{l}\text { Ajdić D, McShan WM, McLaughlin RE, Savić } \\
\text { G, Chang J, Carson MB, Primeaux C, Tian R, } \\
\text { Kenton S, Jia H: Genome sequence of Strep- } \\
\text { tococcus mutans ua159, a cariogenic dental } \\
\text { pathogen. Proc Natl Acad Sci USA 2002; } \\
\text { 99:14434-14439 }\end{array}$ & 1,115 & 652 & 955 & 69.68 & 29 \\
\hline 3 & $\begin{array}{l}\text { Selwitz RH, Ismail Al, Pitts NB: Dental } \\
\text { caries. Lancet 2007; 369:51-59 }\end{array}$ & 905 & 952 & 1,954 & 82.27 & 98 \\
\hline 4 & $\begin{array}{l}\text { Gustafsson BE, Quensel C, Lanke LS, } \\
\text { Lundqvist C, Grahnen H, Bonow B, Krasse } \\
\text { B: The vipeholm dental caries study. The } \\
\text { effect of different levels of carbohydrate } \\
\text { intake on caries activity in } 436 \text { individu- } \\
\text { als observed for five years. Acta Odontol } \\
\text { Scand 1954; } 11: 232-364\end{array}$ & 567 & 323 & 976 & 8.86 & 4 \\
\hline 5 & $\begin{array}{l}\text { Ismail A, Sohn W, Tellez M, Amaya A, Sen } \\
\text { A, Hasson H, Pitts NB: The international } \\
\text { caries detection and assessment system } \\
\text { (ICDAS): An integrated system for meas- } \\
\text { uring dental caries. Community Dent Oral } \\
\text { Epidemiol 2007; 35:170-178 }\end{array}$ & 486 & 497 & 930 & 44.19 & 58 \\
\hline 6 & $\begin{array}{l}\text { Keyes PH: Dental caries in the molar teeth } \\
\text { of rats: II. A method for diagnosing and } \\
\text { scoring several types of lesions simultane- } \\
\text { ously. J Dent Res 1958; } 37: 1088-1099\end{array}$ & 442 & 289 & 518 & 7.37 & 9 \\
\hline 7 & $\begin{array}{l}\text { Featherstone JD: The science and practice } \\
\text { of caries prevention. J Am Dent Assoc } \\
\text { 2000; 131:887-899 }\end{array}$ & 437 & 496 & 1,132 & 24.28 & 24 \\
\hline 8 & $\begin{array}{l}\text { Featherstone JD: Prevention and reversal } \\
\text { of dental caries: Role of low level fluoride. } \\
\text { Community Dent Oral Epidemiol 1999; } \\
27: 31-40\end{array}$ & 428 & 480 & 988 & 22.53 & 36 \\
\hline 9 & $\begin{array}{l}\text { Fitzgerald RJ, Keyes PH: Demonstration of } \\
\text { the etiologic role of streptococci in exper- } \\
\text { imental caries in the hamster. J Am Dent } \\
\text { Assoc 1960; 61:9-19 }\end{array}$ & 427 & 230 & 724 & 7.36 & 2 \\
\hline 10 & $\begin{array}{l}\text { Axelsson P, Lindhe J: Effect of controlled } \\
\text { oral hygiene procedures on caries and peri- } \\
\text { odontal disease in adults. J Clin Periodontol } \\
\text { 1978; 5:133-151 }\end{array}$ & 413 & 342 & 737 & 10.32 & 4 \\
\hline 11 & $\begin{array}{l}\text { Terleckyj B, Willett N, Shockman G: Growth } \\
\text { of several cariogenic strains of oral strep- } \\
\text { tococci in a chemically defined medium. } \\
\text { Infect Immun 1975; 11:649-655 }\end{array}$ & 403 & 230 & 431 & 9.37 & 6 \\
\hline 12 & $\begin{array}{l}\text { Axelsson P, Nyström B, Lindhe J: The } \\
\text { long-term effect of a plaque control } \\
\text { program on tooth mortality, caries and } \\
\text { periodontal disease in adults: Results after } \\
30 \text { years of maintenance. J Clin Periodontol } \\
\text { 2004;31:749-757 }\end{array}$ & 400 & 421 & 816 & 28.57 & 36 \\
\hline
\end{tabular}


Table 1 (continued)

\begin{tabular}{|c|c|c|c|c|c|c|}
\hline Rank & Title of the article & $\begin{array}{l}\text { No. of } \\
\text { citation } \\
\text { (Web of } \\
\text { Science) }\end{array}$ & $\begin{array}{l}\text { No. of } \\
\text { citation } \\
\text { (Scopus) }\end{array}$ & $\begin{array}{l}\text { No. of } \\
\text { citation } \\
\text { (Google } \\
\text { Scholar) }\end{array}$ & $\begin{array}{l}\text { Citation } \\
\text { density }\end{array}$ & CCI 2019 \\
\hline 13 & $\begin{array}{l}\text { Becker MR, Paster BJ, Leys EJ, Moesch- } \\
\text { berger ML, Kenyon SG, Galvin JL, Boches } \\
\text { SK, Dewhirst FE, Griffen AL: Molecular } \\
\text { analysis of bacterial species associated with } \\
\text { childhood caries. J Clin Microbiol 2002; } \\
\text { 40:1001-1009 }\end{array}$ & 400 & 403 & 713 & 25.00 & 25 \\
\hline 14 & $\begin{array}{l}\text { Marthaler T: Changes in dental caries } \\
\text { 1953-2003. Caries Res 2004; 38: } \\
\text { 173-181 }\end{array}$ & 397 & 408 & 866 & 28.35 & 13 \\
\hline 15 & $\begin{array}{l}\text { Takahashi N, Nyvad B: The role of bacteria } \\
\text { in the caries process: Ecological perspec- } \\
\text { tives. J Dent Res } 2011 ; 90: 294-303\end{array}$ & 384 & 397 & 639 & 54.85 & 55 \\
\hline 16 & $\begin{array}{l}\text { Wiegand A, Buchalla W, Attin T: Review } \\
\text { on fluoride-releasing restorative materi- } \\
\text { als-fluoride release and uptake character- } \\
\text { istics, antibacterial activity and influence } \\
\text { on caries formation. Dent Mater 2007; } \\
\text { 23:343-362 }\end{array}$ & 366 & 378 & 655 & 33.27 & 34 \\
\hline 17 & $\begin{array}{l}\text { Beltrán-Aguilar ED, Barker LK, Canto MT, } \\
\text { Dye BA, Gooch BF, Griffin SO, Hyman J, } \\
\text { Jaramillo F, Kingman A, Nowjack-Raymer } \\
\text { R: Surveillance for dental caries, dental } \\
\text { sealants, tooth retention, edentulism, and } \\
\text { enamel fluorosis; united states, 1988-1994 } \\
\text { and 1999-2002. MMWR Surveill Summ } \\
\text { 2005; 54:1-43 }\end{array}$ & 366 & 455 & 728 & 28.15 & 12 \\
\hline 18 & $\begin{array}{l}\text { Van Houte J: Role of micro-organisms in } \\
\text { caries etiology. J Dent Res 1994; 73:672- } \\
681\end{array}$ & 362 & 395 & 803 & 15.08 & 15 \\
\hline 19 & $\begin{array}{l}\text { Tjäderhane L, Larjava H, Sorsa T, Uitto V-J, } \\
\text { Larmas M, Salo T: The activation and func- } \\
\text { tion of host matrix metalloproteinases in } \\
\text { dentin matrix breakdown in caries lesions. J } \\
\text { Dent Res 1998; 77:1622-1629 }\end{array}$ & 356 & 360 & 561 & 17.80 & 18 \\
\hline 20 & $\begin{array}{l}\text { Aas JA, Griffen AL, Dardis SR, Lee AM, Olsen } \\
\text { I, Dewhirst FE, Leys EJ, Paster BJ: Bacteria } \\
\text { of dental caries in primary and permanent } \\
\text { teeth in children and young adults. J Clin } \\
\text { Microbiol 2008; 46:1407-1417 }\end{array}$ & 351 & 337 & 641 & 35.10 & 39 \\
\hline 21 & $\begin{array}{l}\text { Featherstone J, Ten Cate J, Shariati M, } \\
\text { Arends J: Comparison of artificial caries-like } \\
\text { lesions by quantitative microradiography } \\
\text { and microhardness profiles. Caries Res } \\
\text { 1983; 17:385-391 }\end{array}$ & 346 & 342 & 563 & 9.88 & 11 \\
\hline 22 & $\begin{array}{l}\text { Bowen W, Koo H: Biology of Streptococcus } \\
\text { mutans-derived glucosyltransferases: Role } \\
\text { in extracellular matrix formation of cario- } \\
\text { genic biofilms. Caries Res } 2011 ; 45: 69-86\end{array}$ & 340 & 344 & 525 & 48.57 & 49 \\
\hline 23 & $\begin{array}{l}\text { Harris R, Nicoll AD, Adair PM, Pine CM: Risk } \\
\text { factors for dental caries in young children: } \\
\text { A systematic review of the literature. Com- } \\
\text { munity Dent Health } 2004 ; 21: 71-85\end{array}$ & 333 & 360 & 781 & 23.78 & 22 \\
\hline
\end{tabular}

(continued) 
Table 1 (continued)

\begin{tabular}{|c|c|c|c|c|c|c|}
\hline Rank & Title of the article & $\begin{array}{l}\text { No. of } \\
\text { citation } \\
\text { (Web of } \\
\text { Science) }\end{array}$ & $\begin{array}{l}\text { No. of } \\
\text { citation } \\
\text { (Scopus) }\end{array}$ & $\begin{array}{l}\text { No. of } \\
\text { citation } \\
\text { (Google } \\
\text { Scholar) }\end{array}$ & $\begin{array}{l}\text { Citation } \\
\text { density }\end{array}$ & CCI 2019 \\
\hline 24 & $\begin{array}{l}\text { Bagramian RA, Garcia-Godoy F, Volpe } \\
\text { AR: The global increase in dental caries. } \\
\text { A pending public health crisis. Am J dent } \\
2009 ; 22: 3-8\end{array}$ & 330 & 357 & 694 & 36.66 & 31 \\
\hline 25 & $\begin{array}{l}\text { Bratthall D, Hänsel-Petersson G, Sundberg } \\
\text { H: Reasons for the caries decline: What do } \\
\text { the experts believe? Eur J Oral Sci 1996; } \\
\text { 104:416-422 }\end{array}$ & 328 & 377 & 744 & 14.90 & 10 \\
\hline 26 & $\begin{array}{l}\text { Axelsson P, Lindhe J: Effect of controlled } \\
\text { oral hygiene procedures on caries and } \\
\text { periodontal disease in adults: Results after } \\
6 \text { years. J Clin Periodontol 1981; 8:239-248 }\end{array}$ & 304 & 284 & 614 & 8.21 & 8 \\
\hline 27 & $\begin{array}{l}\text { Yamashita Y, Bowen W, Burne R, Kuramitsu } \\
\text { H: Role of the Streptococcus mutans GTF } \\
\text { genes in caries induction in the specif- } \\
\text { ic-pathogen-free rat model. Infect Immun } \\
\text { 1993; 61:3811-3817 }\end{array}$ & 303 & 297 & 388 & 12.12 & 7 \\
\hline 28 & $\begin{array}{l}\text { Näse L, Hatakka K, Savilahti E, Saxelin M, } \\
\text { Pönkä A, Poussa T, Korpela R, Meurman JH: } \\
\text { Effect of long-term consumption of a pro- } \\
\text { biotic bacterium, Lactobacillus rhamnosus } \\
\text { GG, in milk on dental caries and caries risk } \\
\text { in children. Caries Res 2001; 35:412-420 }\end{array}$ & 299 & 314 & 592 & 17.58 & 14 \\
\hline 29 & $\begin{array}{l}\text { Gibbons R, Berman K, Knoettner P, Kapsi- } \\
\text { malis B: Dental caries and alveolar bone } \\
\text { loss in gnotobiotic rats infected with cap- } \\
\text { sule forming streptococci of human origin. } \\
\text { Arch Oral Biol 1966; 11:549-560 }\end{array}$ & 297 & 159 & 401 & 5.71 & 1 \\
\hline 30 & $\begin{array}{l}\text { Krasse B: Human streptococci and exper- } \\
\text { imental caries in hamsters. Arch Oral Biol } \\
\text { 1966; 11:429-414 }\end{array}$ & 286 & 184 & 361 & 5.50 & - \\
\hline 31 & $\begin{array}{l}\text { Marthaler TM, Brunelle J, Downer M, König } \\
\text { K, Truin G, Künzel W, O’Mullane D, Møller } \\
\text { I, von der Fehr F, Vrbic V: The prevalence of } \\
\text { dental caries in Europe 1990-1995. Caries } \\
\text { Res 1996; 30: } \\
\text { 237-255. }\end{array}$ & 281 & 331 & 679 & 12.77 & 6 \\
\hline 32 & $\begin{array}{l}\text { Fejerskov O: Changing paradigms in } \\
\text { concepts on dental caries: consequenc- } \\
\text { es for oral health care. Caries Res 2004; } \\
38: 182-191\end{array}$ & 279 & 306 & 817 & 19.92 & 23 \\
\hline 33 & $\begin{array}{l}\text { Kaste LM, Selwitz RH, Oldakowski RJ, } \\
\text { Brunelle J, Winn DM, Brown LJ: Coronal car- } \\
\text { ies in the primary and permanent dentition } \\
\text { of children and adolescents 1-17 years of } \\
\text { age: United states, 1988-1991. J Dent Res } \\
\text { 1996; 75:631-641 }\end{array}$ & 278 & 323 & 609 & 12.63 & 4 \\
\hline 34 & $\begin{array}{l}\text { Vargas CM, Crall JJ, Schneider DA: Sociode- } \\
\text { mographic distribution of pediatric dental } \\
\text { caries: Nhanes iii, 1988-1994. J Am Dent } \\
\text { Assoc 1998; 129:1229-1238 }\end{array}$ & 277 & 328 & 603 & 13.85 & 4 \\
\hline
\end{tabular}

(continued) 
Table 1 (continued)

\begin{tabular}{|c|c|c|c|c|c|c|}
\hline Rank & Title of the article & $\begin{array}{l}\text { No. of } \\
\text { citation } \\
\text { (Web of } \\
\text { Science) }\end{array}$ & $\begin{array}{l}\text { No. of } \\
\text { citation } \\
\text { (Scopus) }\end{array}$ & $\begin{array}{l}\text { No. of } \\
\text { citation } \\
\text { (Google } \\
\text { Scholar) }\end{array}$ & $\begin{array}{l}\text { Citation } \\
\text { density }\end{array}$ & CCI 2019 \\
\hline 35 & $\begin{array}{l}\text { Lussi A, Imwinkelried S, Pitts N, Longbot- } \\
\text { tom C, Reich E: Performance and repro- } \\
\text { ducibility of a laser fluorescence system for } \\
\text { detection of occlusal caries in vitro. Caries } \\
\text { Res 1999; 33:261-266 }\end{array}$ & 276 & 307 & 533 & 14.52 & 6 \\
\hline 36 & $\begin{array}{l}\text { Gibbons R, Houte J: Dental caries. Annu } \\
\text { Rev Med 1975; 26:121-136 }\end{array}$ & 269 & 158 & 356 & 6.25 & 1 \\
\hline 37 & $\begin{array}{l}\text { Kassebaum N, Bernabé E, Dahiya M, } \\
\text { Bhandari B, Murray C, Marcenes W: Global } \\
\text { burden of untreated caries: a systematic re- } \\
\text { view and metaregression. J Dent Res 2015; } \\
\text { 94:650-658 }\end{array}$ & 267 & 290 & 480 & 89.00 & 86 \\
\hline 38 & $\begin{array}{l}\text { Nyvad B, Machiulskiene V, Bælum V: } \\
\text { Reliability of a new caries diagnostic } \\
\text { system differentiating between active and } \\
\text { inactive caries lesions. Caries Res 1999; } \\
\text { 33:252-260 }\end{array}$ & 262 & 282 & 544 & 13.78 & 19 \\
\hline 39 & $\begin{array}{l}\text { Gibbons R, Banghart S: Synthesis of extra- } \\
\text { cellular dextran by cariogenic bacteria and } \\
\text { its presence in human dental plaque. Arch } \\
\text { Oral Biol } 1967 ; 12: 11-24\end{array}$ & 260 & 125 & 315 & 5.09 & 2 \\
\hline 40 & $\begin{array}{l}\text { Axelsson P, Lindhe J, Nyström B: On the } \\
\text { prevention of caries and periodontal } \\
\text { disease: Results of a 15-year longitudinal } \\
\text { study in adults. J Clin Periodontol 1991; } \\
\text { 18:182-189 }\end{array}$ & 260 & 264 & 557 & 9.62 & 8 \\
\hline 41 & $\begin{array}{l}\text { Petersen PE, Lennon MA: Effective use of } \\
\text { fluorides for the prevention of dental caries } \\
\text { in the 21st century: The WHO approach. } \\
\text { Community Dent Oral Epidemiol 2004; } \\
\text { 32:319-321 }\end{array}$ & 258 & 212 & 511 & 18.42 & 19 \\
\hline 42 & $\begin{array}{l}\text { Fried D, Xie J, Shafi S, Featherstone JD, } \\
\text { Breunig T, Le CQ: Imaging caries lesions } \\
\text { and lesion progression with polarization } \\
\text { sensitive optical coherence tomography. J } \\
\text { Biomed Opt 2002; 7:618-628 }\end{array}$ & 258 & 276 & 487 & 16.12 & 7 \\
\hline 43 & $\begin{array}{l}\text { Axelsson P, Lindhe J: The effect of a preven- } \\
\text { tive programme on dental plaque, gingi- } \\
\text { vitis and caries in schoolchildren. Results } \\
\text { after one and two years. J Clin Periodontol } \\
\text { 1974; 1:126-138 }\end{array}$ & 257 & 222 & 430 & 5.84 & 2 \\
\hline 44 & $\begin{array}{l}\text { Tanzer JM, Livingston J, Thompson AM: The } \\
\text { microbiology of primary dental caries in } \\
\text { humans. J Dent Educ 2001; 65:1028-1037 }\end{array}$ & 256 & 268 & 541 & 15.05 & 13 \\
\hline 45 & $\begin{array}{l}\text { Sakanaka S, Kim M, Taniguchi M, Yamamo- } \\
\text { to T: Antibacterial substances in Japanese } \\
\text { green tea extract against Streptococcus } \\
\text { mutans, a cariogenic bacterium. Agric Biol } \\
\text { Chem 1989; 53:2307-2311 }\end{array}$ & 252 & 273 & 497 & 8.68 & - \\
\hline 46 & $\begin{array}{l}\text { Nakajima M, Sano H, Burrow M, Tagami J, } \\
\text { Yoshiyama M, Ebisu S, Ciucchi B, Russell } \\
\text { C, Pashley DH: Tensile bond strength and } \\
\text { SEM evaluation of caries-affected dentin } \\
\text { using dentin adhesives. J Dent Res 1995; } \\
\text { 74:1679-1688 }\end{array}$ & 251 & 258 & 436 & 10.91 & 2 \\
\hline
\end{tabular}


Table 1 (continued)

\begin{tabular}{|c|c|c|c|c|c|c|}
\hline Rank & Title of the article & $\begin{array}{l}\text { No. of } \\
\text { citation } \\
\text { (Web of } \\
\text { Science) }\end{array}$ & $\begin{array}{l}\text { No. of } \\
\text { citation } \\
\text { (Scopus) }\end{array}$ & $\begin{array}{l}\text { No. of } \\
\text { citation } \\
\text { (Google } \\
\text { Scholar) }\end{array}$ & $\begin{array}{l}\text { Citation } \\
\text { density }\end{array}$ & CCI 2019 \\
\hline 47 & $\begin{array}{l}\text { Keyes PH: The infectious and transmissi- } \\
\text { ble nature of experimental dental caries: } \\
\text { Findings and implications. Arch Oral Biol } \\
1960 ; 1: 304-320\end{array}$ & 250 & 161 & 580 & 4.31 & 3 \\
\hline 48 & $\begin{array}{l}\text { Moreno EC, Kresak M, Zahradnik RT: Fluor- } \\
\text { idated hydroxyapatite solubility and caries } \\
\text { formation. Nature } 1974 ; 247: 64-65\end{array}$ & 244 & 237 & 314 & 5.54 & 10 \\
\hline 49 & $\begin{array}{l}\text { Lussi A, Megert B, Longbottom C, Reich } \\
\text { E, Francescut P: Clinical performance of a } \\
\text { laser fluorescence device for detection of } \\
\text { occlusal caries lesions. Eur J Oral Sci 2001; } \\
\text { 109:14-19 }\end{array}$ & 240 & 261 & 487 & 14.11 & 7 \\
\hline 50 & $\begin{array}{l}\text { Loesche W, Rowan J, Straffon L, Loos P: } \\
\text { Association of Streptococcus mutans with } \\
\text { human dental decay. Infec Immun 1975; } \\
\text { 11:1252-1260 }\end{array}$ & 240 & 187 & 394 & 5.58 & 1 \\
\hline 51 & $\begin{array}{l}\text { Leme AP, Koo H, Bellato C, Bedi G, Cury } \\
\text { J: The role of sucrose in cariogenic dental } \\
\text { biofilm formation-new insight. J Dent Res } \\
2006 ; 85: 878-887\end{array}$ & 239 & 249 & 431 & 19.91 & 20 \\
\hline 52 & $\begin{array}{l}\text { Mjör IA, Toffentti F: Secondary caries: A } \\
\text { literature review with case reports. Quin- } \\
\text { tessence Int 2000; 31:165-179 }\end{array}$ & 238 & 246 & 435 & 13.22 & 7 \\
\hline 53 & $\begin{array}{l}\text { Chaussain-Miller C, Fioretti F, Goldberg M, } \\
\text { Menashi S: The role of matrix metallopro- } \\
\text { teinases (MMPs) in human caries. J Dent } \\
\text { Res 2006; 85:22-32 }\end{array}$ & 237 & 240 & 406 & 19.75 & 17 \\
\hline 54 & $\begin{array}{l}\text { Nyvad B, Kilian M: Comparison of the initial } \\
\text { streptococcal microflora on dental enamel } \\
\text { in caries-active and in caries-inactive indi- } \\
\text { viduals. Caries Res } 1990 ; 24: 267-272\end{array}$ & 235 & 216 & 356 & 8.39 & 6 \\
\hline 55 & $\begin{array}{l}\text { Moreno E, Kresak M, Zahradnik R: Phys- } \\
\text { icochemical aspects of fluoride-apatite } \\
\text { systems relevant to the study of dental } \\
\text { caries. Caries Res } 1977 ; 11: 142-171\end{array}$ & 230 & 215 & 283 & 5.60 & 2 \\
\hline 56 & $\begin{array}{l}\text { Aoki A, Ishikawa I, Yamada T, Otsuki M, } \\
\text { Watanabe H, Tagami J, Ando Y, Yamamo- } \\
\text { to H: Comparison between er: Yag laser } \\
\text { and conventional technique for root } \\
\text { caries treatment in vitro. J Dent Res 1998; } \\
\text { 77:1404-1414 }\end{array}$ & 227 & 246 & 399 & 11.36 & 5 \\
\hline 57 & $\begin{array}{l}\text { Guggenheim B, Schroeder H: Biochemical } \\
\text { and morphological aspects of extracellular } \\
\text { polysaccharides produced by cariogenic } \\
\text { streptococci. Helv Odontol Acta 1967; } \\
\text { 11:131-152 }\end{array}$ & 224 & 111 & 287 & 4.39 & - \\
\hline 58 & $\begin{array}{l}\text { Moynihan P, Kelly S: Effect on caries of } \\
\text { restricting sugars intake: Systematic review } \\
\text { to inform who guidelines. J Dent Res 2014; } \\
93: 8-18\end{array}$ & 222 & 249 & 466 & 55.50 & 57 \\
\hline 59 & $\begin{array}{l}\text { Munson M, Banerjee A, Watson T, Wade } \\
\text { W: Molecular analysis of the microflora as- } \\
\text { sociated with dental caries. J Clin Microbiol } \\
\text { 2004; 42:3023-3029 }\end{array}$ & 221 & 220 & 370 & 15.78 & 14 \\
\hline
\end{tabular}

(continued) 
Table 1 (continued)

\begin{tabular}{|c|c|c|c|c|c|c|}
\hline Rank & Title of the article & $\begin{array}{l}\text { No. of } \\
\text { citation } \\
\text { (Web of } \\
\text { Science) }\end{array}$ & $\begin{array}{l}\text { No. of } \\
\text { citation } \\
\text { (Scopus) }\end{array}$ & $\begin{array}{l}\text { No. of } \\
\text { citation } \\
\text { (Google } \\
\text { Scholar) }\end{array}$ & $\begin{array}{l}\text { Citation } \\
\text { density }\end{array}$ & CCI 2019 \\
\hline 60 & $\begin{array}{l}\text { Bratthall D: Introducing the significant } \\
\text { caries index together with a proposal for } \\
\text { a new global oral health goal for } 12 \text {-year- } \\
\text { olds. Int Dent J } 2000 ; 50: 378-384\end{array}$ & 216 & 219 & 576 & 12.00 & 10 \\
\hline 61 & $\begin{array}{l}\text { Sheiham A: Dental caries affects body } \\
\text { weight, growth and quality of life in pre- } \\
\text { school children. Br Dent J 2006; 201:625- } \\
626\end{array}$ & 215 & 224 & 479 & 17.91 & 21 \\
\hline 62 & $\begin{array}{l}\text { Byun R, Nadkarni MA, Chhour K-L, Martin } \\
\text { FE, Jacques NA, Hunter N: Quantitative } \\
\text { analysis of diverse lactobacillus species } \\
\text { present in advanced dental caries. J Clin } \\
\text { Microbiol 2004; 42:3128-3136 }\end{array}$ & 214 & 207 & 370 & 15.28 & 10 \\
\hline 63 & $\begin{array}{l}\text { Fejerskov O, Thylstrup A, Larsen MJ: Ra- } \\
\text { tional use of fluorides in caries prevention: } \\
\text { A concept based on possible cariostatic } \\
\text { mechanisms. Acta Odontol Scand 1981; } \\
\text { 39:241-249 }\end{array}$ & 214 & 202 & 378 & 5.78 & 6 \\
\hline 64 & $\begin{array}{l}\text { Shi X-Q, Welander U, Angmar-Månsson } \\
\text { B: Occlusal caries detection with KaVo } \\
\text { DIAGNOdent and radiography: An in vitro } \\
\text { comparison. Caries Res 2000; 34:151-158 }\end{array}$ & 207 & 233 & 376 & 11.50 & 6 \\
\hline 65 & $\begin{array}{l}\text { Filstrup SL, Briskie D, Da Fonseca M, } \\
\text { Lawrence L, Wandera A, Inglehart MR: } \\
\text { Early childhood caries and quality of life: } \\
\text { Child and parent perspectives. Pediatr Dent } \\
\text { 2003; 25:431-440 }\end{array}$ & 206 & 219 & 449 & 13.73 & 15 \\
\hline 66 & $\begin{array}{l}\text { Dreizen S, Brown LR, Daly TE, Drane JB: } \\
\text { Prevention of xerostomia-related dental } \\
\text { caries in irradiated cancer patients. J Dent } \\
\text { Res } 1977 ; 56: 99-104\end{array}$ & 206 & 208 & 319 & 5.02 & 4 \\
\hline 67 & $\begin{array}{l}\text { Takahashi N, Nyvad B: Caries ecology } \\
\text { revisited: Microbial dynamics and the caries } \\
\text { process. Caries Res 2008; 42:409-418 }\end{array}$ & 204 & 220 & 450 & 20.40 & 17 \\
\hline 68 & $\begin{array}{l}\text { Levitch L, Bader J, Shugars D, Heymann } \\
\text { H: Non-carious cervical lesions. J of Dent } \\
\text { 1994; 22:195-207 }\end{array}$ & 201 & 202 & 447 & 8.37 & 4 \\
\hline 69 & $\begin{array}{l}\text { Alaluusua S, Renkonen OV: Streptococcus } \\
\text { mutans establishment and dental caries } \\
\text { experience in children from } 2 \text { to } 4 \text { years } \\
\text { old. Eur J Oral Sci 1983; 91:453-457 }\end{array}$ & 200 & 111 & 405 & 5.71 & 1 \\
\hline 70 & $\begin{array}{l}\text { Van Nieuw Amerongen A, Bolscher JG, } \\
\text { Veerman EC: Salivary proteins: Protective } \\
\text { and diagnostic value in cariology? Caries } \\
\text { Res 2004; 38:247-253 }\end{array}$ & 199 & 246 & 458 & 14.21 & 11 \\
\hline 71 & $\begin{array}{l}\text { Lukacs JR, Largaespada LL: Explaining sex dif- } \\
\text { ferences in dental caries prevalence: Saliva, } \\
\text { hormones, and “life-history” etiologies. Am } \\
\text { J Hum Biol 2006; 18:540-555 }\end{array}$ & 198 & 203 & 423 & 16.5 & 15 \\
\hline 72 & $\begin{array}{l}\text { Pitts N: ICDAS-an international system for } \\
\text { caries detection and assessment being } \\
\text { developed to facilitate caries epidemiology, } \\
\text { research and appropriate clinical man- } \\
\text { agement. Community Dent Health 2004; } \\
\text { 21:193-198 }\end{array}$ & 198 & 225 & 405 & 14.14 & 18 \\
\hline
\end{tabular}


Table 1 (continued)

\begin{tabular}{|c|c|c|c|c|c|c|}
\hline Rank & Title of the article & $\begin{array}{l}\text { No. of } \\
\text { citation } \\
\text { (Web of } \\
\text { Science) }\end{array}$ & $\begin{array}{l}\text { No. of } \\
\text { citation } \\
\text { (Scopus) }\end{array}$ & $\begin{array}{l}\text { No. of } \\
\text { citation } \\
\text { (Google } \\
\text { Scholar) }\end{array}$ & $\begin{array}{l}\text { Citation } \\
\text { density }\end{array}$ & CCI 2019 \\
\hline 73 & $\begin{array}{l}\text { Fusayama T: Two layers of carious dentin; } \\
\text { diagnosis and treatment. Oper Dent 1979; } \\
\text { 4:63-70 }\end{array}$ & 198 & 185 & 377 & 5.07 & 8 \\
\hline 74 & $\begin{array}{l}\text { Edwardsson S: Characteristics of caries-in- } \\
\text { ducing human streptococci resembling } \\
\text { Streptococcus mutans. Arch Oral Biol 1968; } \\
\text { 13:637-646 }\end{array}$ & 197 & 111 & 261 & 3.94 & 1 \\
\hline 75 & $\begin{array}{l}\text { Ahola A, Yli-Knuuttila H, Suomalainen } \\
\text { T, Poussa T, Ahlström A, Meurman JH, } \\
\text { Korpela R: Short-term consumption of } \\
\text { probiotic-containing cheese and its effect } \\
\text { on dental caries risk factors. Arch Oral Biol } \\
\text { 2002; 47:799-804 }\end{array}$ & 196 & 203 & 393 & 12.25 & 7 \\
\hline 76 & $\begin{array}{l}\text { De Stoppelaar J, Van Houte J, Dirks OB: } \\
\text { The relationship between extracellular } \\
\text { polysaccharide-producing streptococci } \\
\text { and smooth surface caries in 13-year-old } \\
\text { children. Caries Res 1969; 3:190-199 }\end{array}$ & 195 & 179 & 331 & 3.97 & - \\
\hline 77 & $\begin{array}{l}\text { Petersen PE: Sociobehavioural risk factors } \\
\text { in dental caries-international perspectives. } \\
\text { Community Dent Oral Epidemiol 2005; } \\
33: 274-279\end{array}$ & 193 & 196 & 479 & 14.84 & 6 \\
\hline 78 & $\begin{array}{l}\text { Kleinberg I: A mixed-bacteria ecological } \\
\text { approach to understanding the role of the } \\
\text { oral bacteria in dental caries causation: An } \\
\text { alternative to Streptococcus mutans and the } \\
\text { specific-plaque hypothesis. Crit Rev Oral Biol } \\
\text { Med 2002; 13:108-125 }\end{array}$ & 192 & 205 & 409 & 12.00 & 11 \\
\hline 79 & $\begin{array}{l}\text { Casamassimo PS, Thikkurissy S, Edelstein BL, } \\
\text { Maiorini E: Beyond the DMFT: The human } \\
\text { and economic cost of early childhood caries. J } \\
\text { Am Dent Assoc 2009; 140:650-657 }\end{array}$ & 191 & 207 & 377 & 21.22 & 17 \\
\hline 80 & $\begin{array}{l}\text { Köhler B, Andréen I, Jonsson B: The earlier } \\
\text { the colonization by mutans streptococci, } \\
\text { the higher the caries prevalence at } 4 \text { years } \\
\text { of age. Oral Microbiol Immunol 1988; } \\
\text { 3:14-17 }\end{array}$ & 190 & 207 & 379 & 6.33 & 1 \\
\hline 81 & $\begin{array}{l}\text { Loesche W, Syed S: Predominant cultivable } \\
\text { flora of carious plaque and carious dentine. } \\
\text { Caries Res 1973; 7:201-216 }\end{array}$ & 189 & 159 & 242 & 4.20 & - \\
\hline 82 & $\begin{array}{l}\text { Beighton D: The complex oral microflora } \\
\text { of high-risk individuals and groups and its } \\
\text { role in the caries process. Community Dent } \\
\text { Oral Epidemiol 2005; 33:248-255 }\end{array}$ & 187 & 187 & 375 & 14.38 & 12 \\
\hline 83 & $\begin{array}{l}\text { Burne RA, Marquis RE: Alkali production by } \\
\text { oral bacteria and protection against dental } \\
\text { caries. FEMS Microbiol Lett 2000; 193:1-6 }\end{array}$ & 186 & 183 & 295 & 10.33 & 16 \\
\hline 84 & $\begin{array}{l}\text { Makinen K, Bennett C, Hujoel P, Isokan- } \\
\text { gas P, Isotupa K, Pape Jr H, Makinen P: } \\
\text { Xylitol chewing gums and caries rates: A } \\
\text { 40-month cohort study. J Dent Res 1995; } \\
\text { 74:1904-1913 }\end{array}$ & 185 & 199 & 368 & 8.04 & 2 \\
\hline 85 & $\begin{array}{l}\text { Zinner DD, Jablon JM, Aran AP, Saslaw MS: } \\
\text { Experimental caries induced in animals by } \\
\text { streptococci of human origin. Exp Biol Med } \\
\text { 1965; } 118: 766-770\end{array}$ & 185 & 93 & 256 & 3.49 & 1 \\
\hline
\end{tabular}

(continued) 
Table 1 (continued)

\begin{tabular}{|c|c|c|c|c|c|c|}
\hline Rank & Title of the article & $\begin{array}{l}\text { No. of } \\
\text { citation } \\
\text { (Web of } \\
\text { Science) }\end{array}$ & $\begin{array}{l}\text { No. of } \\
\text { citation } \\
\text { (Scopus) }\end{array}$ & $\begin{array}{l}\text { No. of } \\
\text { citation } \\
\text { (Google } \\
\text { Scholar) }\end{array}$ & $\begin{array}{l}\text { Citation } \\
\text { density }\end{array}$ & CCI 2019 \\
\hline 86 & $\begin{array}{l}\text { Yoshiyama M, Tay F, Doi J, Nishitani Y, } \\
\text { Yamada T, Itou K, Carvalho R, Nakajima } \\
\text { M, Pashley D: Bonding of self-etch and to- } \\
\text { tal-etch adhesives to carious dentin. J Dent } \\
\text { Res 2002; 81:556-560 }\end{array}$ & 184 & 181 & 325 & 11.50 & 8 \\
\hline 87 & $\begin{array}{l}\text { Marshall TA, Levy SM, Broffitt B, Warren } \\
\text { JJ, Eichenberger-Gilmore JM, Burns TL, } \\
\text { Stumbo PJ: Dental caries and beverage } \\
\text { consumption in young children. Pediatrics } \\
\text { 2003; 112:e184-e191 }\end{array}$ & 183 & 215 & 417 & 12.20 & 7 \\
\hline 88 & $\begin{array}{l}\text { Hillson S: Recording dental caries in archae- } \\
\text { ological human remains. Int J Osteoarchae- } \\
\text { ol 2001; 11:249-289 }\end{array}$ & 183 & 191 & 374 & 10.76 & - \\
\hline 89 & $\begin{array}{l}\text { Brunelle J, Carlos J: Recent trends in dental } \\
\text { caries in us children and the effect of water } \\
\text { fluoridation. J Dent Res 1990; 69:723-727 }\end{array}$ & 181 & 180 & 410 & 6.46 & - \\
\hline 90 & $\begin{array}{l}\text { Brännström M: The hydrodynamic theory } \\
\text { of dentinal pain: Sensation in preparations, } \\
\text { caries, and the dentinal crack syndrome. J } \\
\text { Endod 1986; } 12: 453-457\end{array}$ & 180 & 186 & 402 & 5.65 & 6 \\
\hline 91 & $\begin{array}{l}\text { Narvai PC, Frazao P, Roncalli AG, Antunes J: } \\
\text { Dental caries in Brazil: Decline, polarization, } \\
\text { inequality and social exclusion. Rev Panam } \\
\text { Salud Publica 2006; 19:385-393 }\end{array}$ & 179 & 156 & 126 & 14.91 & 2 \\
\hline 92 & $\begin{array}{l}\text { Kidd EAM, Fejerskov O: What constitutes } \\
\text { dental caries? Histopathology of carious } \\
\text { enamel and dentin related to the action } \\
\text { of cariogenic biofilms. J Dent Res 2004; } \\
\text { 83:35-38 }\end{array}$ & 178 & 228 & 580 & 12.71 & 15 \\
\hline 93 & $\begin{array}{l}\text { Årtun J, Brobakken BO: Prevalence of } \\
\text { carious white spots after orthodontic treat- } \\
\text { ment with multibonded appliances. Eur J } \\
\text { Orthod 1986; 8:229-234 }\end{array}$ & 178 & 170 & 372 & 5.56 & 7 \\
\hline 94 & $\begin{array}{l}\text { Larsen M: The nature of early caries lesions } \\
\text { in enamel. J Dent Res 1986; 65:1030-1031 }\end{array}$ & 176 & 164 & 346 & 5.50 & 4 \\
\hline 95 & $\begin{array}{l}\text { Michalek SM, Mestecky J, Arnold R, Bozzo L: } \\
\text { Ingestion of Streptococcus mutans induces } \\
\text { secretory immunoglobulin A and caries } \\
\text { immunity. Science 1976; 192:1238-1240 }\end{array}$ & 176 & 94 & 235 & 4.19 & 1 \\
\hline 96 & $\begin{array}{l}\text { Von der Fehr FR, Loe H, Theilade E: Exper- } \\
\text { imental caries in man. Caries Res 1970; } \\
4: 131-148\end{array}$ & 176 & 172 & 297 & 3.66 & 2 \\
\hline 97 & $\begin{array}{l}\text { Fitzgerald R, Jordan H, Stanley H, Poole W, } \\
\text { Bowler A: Experimental caries and gingival } \\
\text { pathologic changes in the gnotobiotic rat. J } \\
\text { Dent Res 1960; 39:923-935 }\end{array}$ & 176 & 89 & 256 & 3.03 & - \\
\hline 98 & $\begin{array}{l}\text { Burt BA, Pai S: Sugar consumption and } \\
\text { caries risk: A systematic review. J Dent Educ } \\
\text { 2001; 65:1017-1023 }\end{array}$ & 175 & 188 & 407 & 10.29 & 5 \\
\hline 99 & $\begin{array}{l}\text { Mitchell TJ: The pathogenesis of strep- } \\
\text { tococcal infections: From tooth decay } \\
\text { to meningitis. Nat Rev Microbiol 2003; } \\
\text { 1:219-230 }\end{array}$ & 175 & 169 & 287 & 11.66 & 12 \\
\hline 100 & $\begin{array}{l}\text { De Jong EdJ, Sundström F, Westerling H, } \\
\text { Tranaeus S, Ten Bosch J, Angmar-Månsson } \\
\text { B: A new method for in vivo quantification } \\
\text { of changes in initial enamel caries with laser } \\
\text { fluorescence. Caries Res 1995; } 29: 2-7\end{array}$ & 174 & 192 & 212 & 7.56 & 4 \\
\hline
\end{tabular}

Abbreviation: $\mathrm{CCl}$, current citation index. 


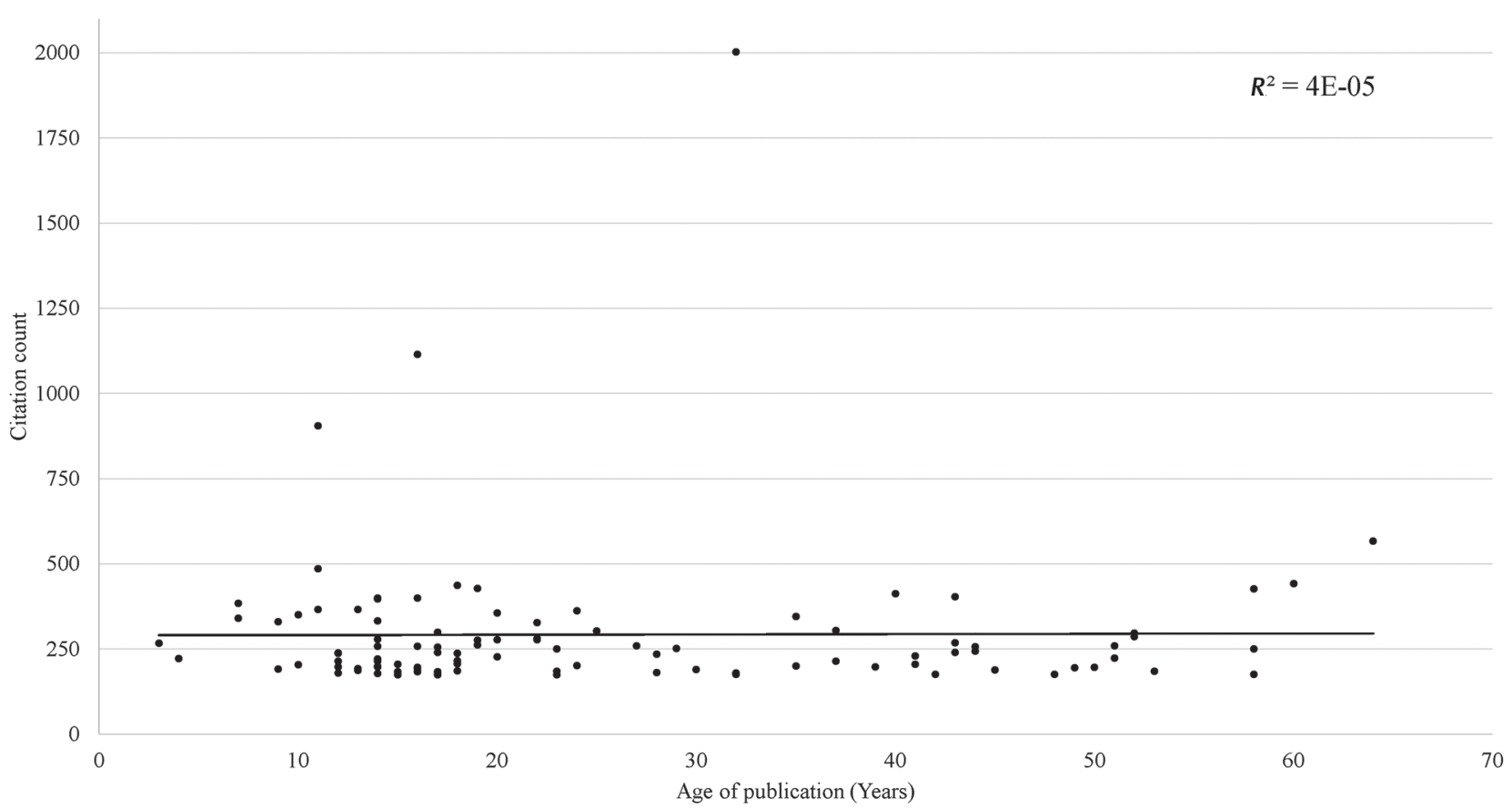

Fig. 1 Association of citation count with age of publication.

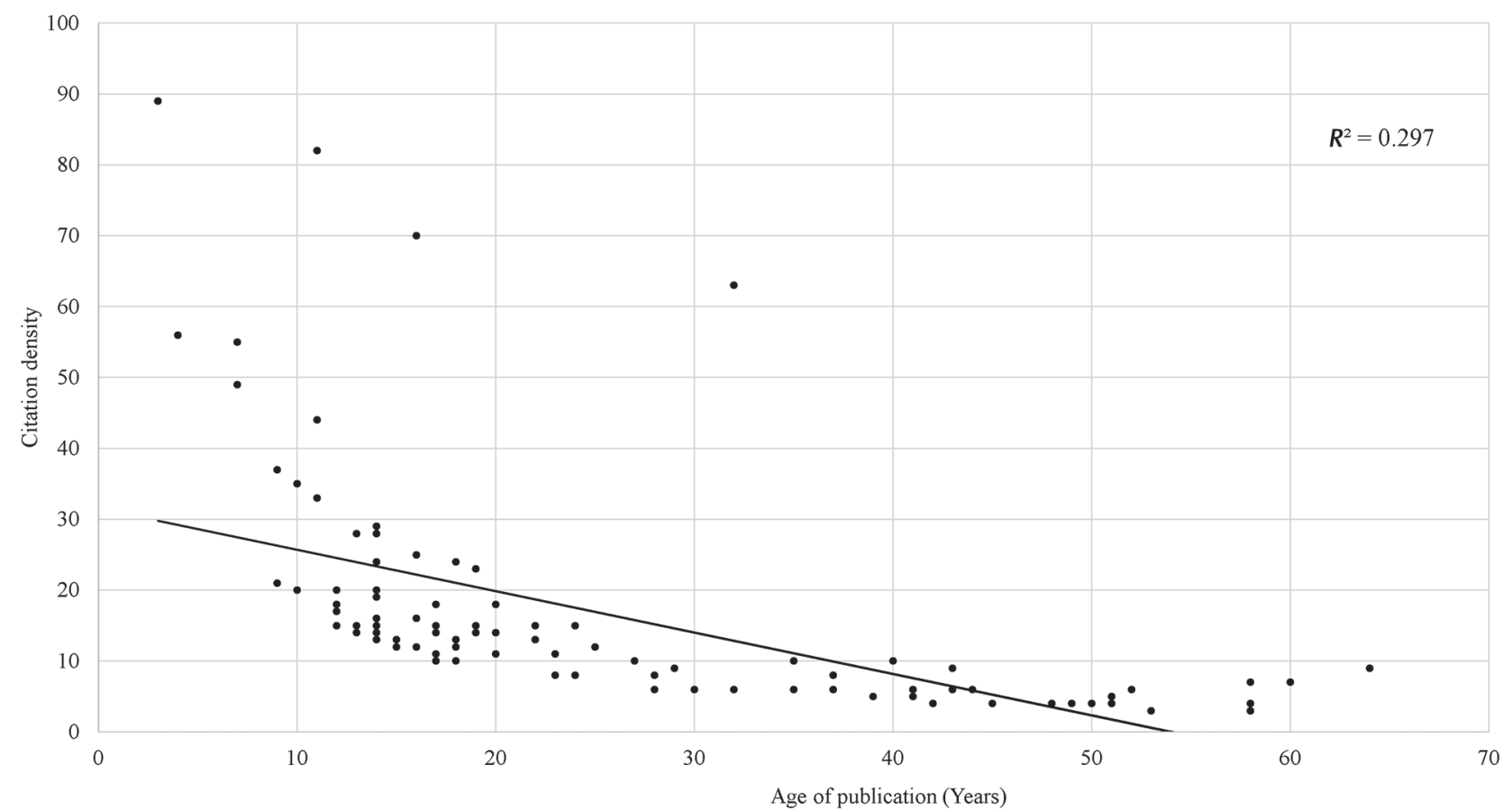

Fig. 2 Change of citation density with age of publication.

Where the keywords are presented as cluster of nodes and the node size represents the frequency of usage of a certain keyword. The size of the node is directly proportional to the usage frequency of a keyword. The thickness of the edges between two keyword nodes represents the closeness of interactions. The node color of the keywords represents their cluster.

\section{Discussion}

The current study focused on the identification and analysis of the top 100 "classics" on dental caries and to highlight the change in current trends, centers of excellence in caries research, dominant types of methodology, and technological developments made over time. In the research 


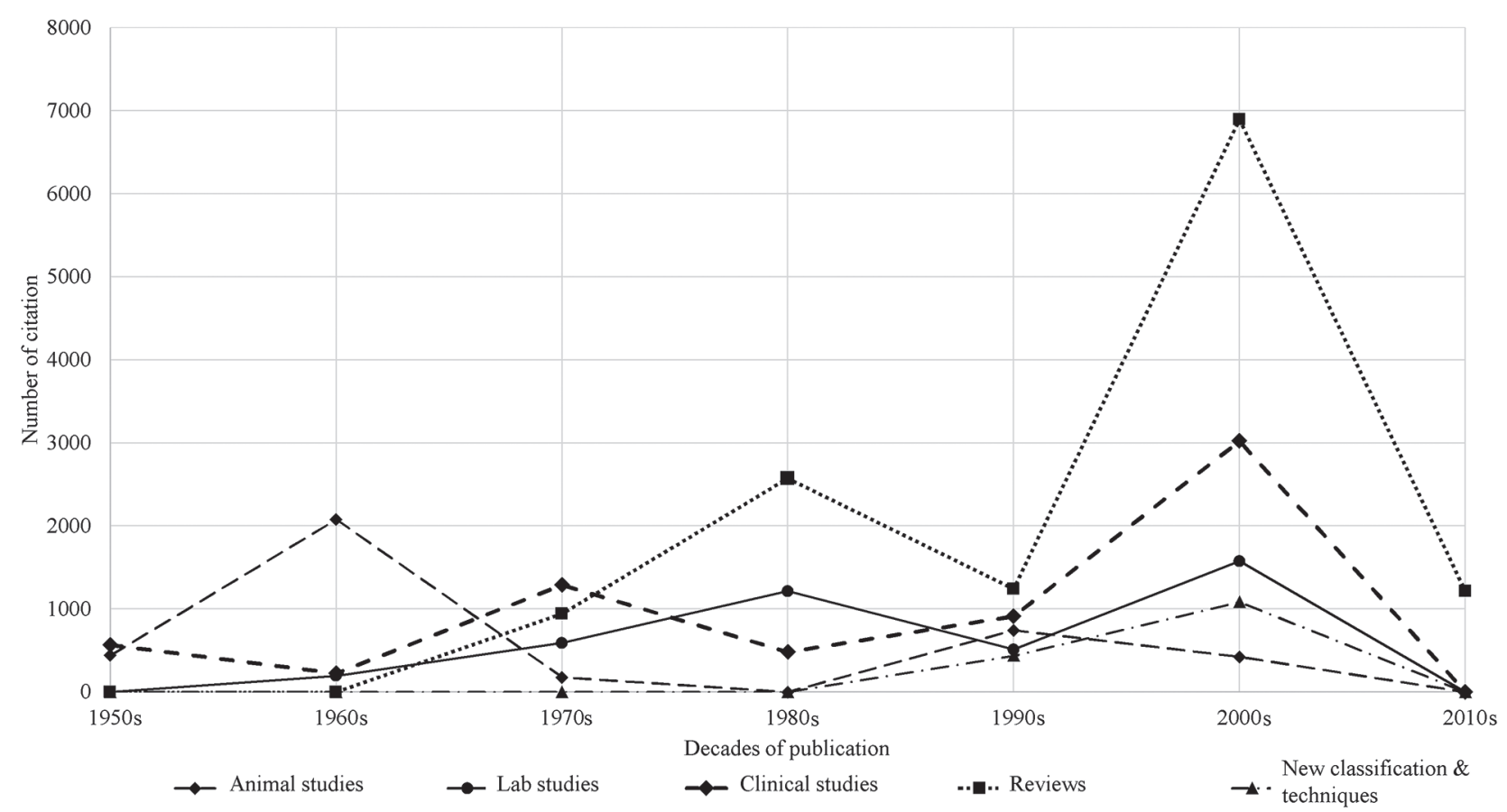

Fig. 3 The time trends in the top 100 most cited caries research articles according to their categories.

field, a publication cited 100 or more times is considered as a "classic" article. ${ }^{27}$ Therefore, all articles included in this study can be termed as "classic" in the field of dental caries. WoS was used as a benchmark database as it is capable of measuring the number of citations since 1945 until date. ${ }^{17}$ Upon cross-matching the citation counts of "classic" publications with Scopus and GS, a considerable fluctuation was evident which varied between 89 to 952 (Scopus), and it ranged between 126 to 1,954 (GS). The evident fluctuation in the citation counts among different databases emphasizes the role of database selection in scientometry. The chance of duplicate article was not expected as only one database was utilized. Nevertheless, it is noteworthy that only citations from 1996 onwards are measured by Scopus. GS includes in its list different forms of cited materials including Web pages, thesis/dissertations/notes, etc., which can provide misleading results while performing an assessment of the most often cited scientific articles in peer-reviewed journals. ${ }^{28}$

Publication age tends to increase the number of citations for any field regardless of their journal impact. ${ }^{29}$ Nevertheless, this assumption is not supported by the current study as $48 \%$ publications were from 2000 to 2015 and 52\% were from 1954 and 1999. Although recently published articles (past 15 years) have a lower probability of making it to the "classic" list, ${ }^{15} 29$ publications were included from 2004 to 2018 . This finding emphasizes the relevance, quality, and impact of the topic of a publication on the clinical practice and research. Recently published articles (after 2015) have also been highly cited; however, it is still too early to foresee how the publication age influences the citation count of these publications.

In addition to current study, other bibliometric studies have documented that authors from Africa, South America, Asia, and the Middle East whether being the corresponding or first authors did not make a major contribution which could be counted toward the "classic" articles. ${ }^{30-33}$ Possible explanations might include language barriers, gaps in professional networking, conducting research, and limited information access. The findings of current study identify a need to turn the focus of caries research toward developing countries where it is more widespread. ${ }^{34}$ The United Nations Organization and the World Health Organization could play a key role in promoting these health care developments.

A total of 301 authors contributed to these "classic" articles. Most of the authors contributed as the first author and the corresponding author simultaneously. J.D.B. Featherstone contributed the most as the corresponding author in three articles. A single author wrote 21 articles and only 2 authors contributed in another 32 articles. Interestingly, N.B. Pitts contributed as the last author of two of the top five "classic" articles which were written by the same author.

The United States have ample financial resources, a larger scientific population, and active researchers which explains its significant contribution to the field of dental caries and explains its greater contribution to the list of top 100 "clas-

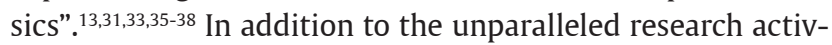
ity of the United States, authors have shown a tendency to favor citation of publication originating from within the United States. ${ }^{39}$ It is interesting to note that 35 out of 45 articles originating from the United States were published in the journals of origin other than the United States. The Scandinavian countries despite their smaller population size made considerable contributions $(n=21)$.

In research, the highest quality of evidence is extracted from randomized controlled trials (RCTs) and presented in systematic reviews which are then subjected to meta-analyses to form an evidence base. ${ }^{40}$ The current study identified 13 RCTs and 4 
Table 2 List of journals which published the top 100 "classic" articles on caries research

\begin{tabular}{|c|c|c|c|c|c|}
\hline Journal name & $\begin{array}{l}\text { No. of } \\
\text { publication }\end{array}$ & $\begin{array}{l}\text { Impact } \\
\text { factor }\end{array}$ & Journal name & $\begin{array}{l}\text { No. of } \\
\text { publication }\end{array}$ & $\begin{array}{l}\text { Impact } \\
\text { factor }\end{array}$ \\
\hline Journal of Dental Research & 19 & 5.383 & Caries Research & 17 & 2.188 \\
\hline Archives of Oral Biology & 6 & 2.050 & $\begin{array}{l}\text { Community Dentistry and Oral } \\
\text { Epidemiology }\end{array}$ & 5 & 1.992 \\
\hline $\begin{array}{l}\text { Journal of the American Dental } \\
\text { Association }\end{array}$ & 4 & 2.486 & Journal of Clinical Periodontology & 5 & 4.046 \\
\hline Journal of Clinical Microbiology & 4 & 4.054 & European Journal of Oral Sciences & 3 & 1.655 \\
\hline Infection and Immunity & 3 & 3.256 & Community Dental Health & 2 & 0.956 \\
\hline Acta Odontologica Scandinavica & 2 & 1.522 & Journal of Dental Education & 2 & 1.102 \\
\hline The Lancet & 1 & 53.254 & Nature & 1 & 41.577 \\
\hline Science & 1 & 41.058 & Nature Reviews Microbiology & 1 & 31.851 \\
\hline Annual Review of Medicine & 1 & 14.970 & $\begin{array}{l}\text { Microbiology and Molecular Biology } \\
\text { Reviews }\end{array}$ & 1 & 13.439 \\
\hline $\begin{array}{l}\text { MMWR: Morbidity and Mortality } \\
\text { Weekly Report }\end{array}$ & 1 & 12.888 & $\begin{array}{l}\text { Proceedings of the National Academy } \\
\text { of Sciences of the United States of } \\
\text { America }\end{array}$ & 1 & 9.504 \\
\hline Pediatrics & 1 & 5.515 & Dental Materials & 1 & 4.039 \\
\hline Journal of dentistry & 1 & 3.770 & Journal of Endodontics & 1 & 2.886 \\
\hline $\begin{array}{l}\text { Oral Microbiology and Immunology } \\
\text { (Molecular Oral Microbiology) }\end{array}$ & 1 & 2.853 & Experimental Biology and Medicine & 1 & 2.413 \\
\hline Journal of Biomedical Optics & 1 & 2.367 & Operative Dentistry & 1 & 2.130 \\
\hline European Journal of Orthodontics & 1 & 2.033 & Pediatric Dental Journal & 1 & 1.947 \\
\hline FEMS Microbiology Letters & 1 & 1.735 & American Journal of Human Biology & 1 & 1.575 \\
\hline $\begin{array}{l}\text { International Journal of Osteoarchae- } \\
\text { ology }\end{array}$ & 1 & 1.432 & International Dental Journal & 1 & 1.389 \\
\hline British Dental Journal & 1 & 1.274 & Agricultural and Biological Chemistry & 1 & 1.255 \\
\hline Helvetica Odontologica Acta & 1 & 1.209 & Quintessence International & 1 & 1.088 \\
\hline $\begin{array}{l}\text { Pan American Journal of Public } \\
\text { Health }\end{array}$ & 1 & 0.784 & American Journal of Dentistry & 1 & 0.760 \\
\hline
\end{tabular}

systematic reviews which were included as "classic" articles. It is noteworthy to mention that the Cochrane reviews could not secure a position in the current study although they are internationally recognized as the highest level of evidence base. ${ }^{34} \mathrm{~A}$ possible explanation of this exclusion is the lower number of citations received by Cochrane reviews is that WoS covers the updated version of Cochrane reviews which started in 2005 and have not yet gained significant age of publications. ${ }^{34}$ Although narrative reviews are classified as a lower level of evidence, 29 review-type publications made to the list of "classic" articles. The presence of so many reviews (36\%) signifies the preference of several authors in compiling existing knowledge and information on the topics within the field of caries for the advantage of coresearchers and readers. With the changing trends, evidence-based dentistry has gained significant importance, whereas the current study indicates that the most often cited articles had lower levels of evidence and not necessarily the greatest scientific importance ${ }^{41}$; this finding has been previously documented. ${ }^{13,15}$

High impact factor journals such as the The Lancet, Nature, Science, Annual Review of Medicine, Journal of Dental Research, and Caries Research have published most of the "classic" publications. An inclination toward publishing in influential journals was noted which follows Bradford's law, that "most researchers secure their citations from a few specific core journals." 42 Publication by these authors in other journals may result in reduction of impact of their publication. Journal of Dental Research, Journal of Clinical Periodontology, and Caries Research are identified as the specific nucleus journals of research related to the caries research.

Keywords are the most important component of a research paper in terms of accessibility. Keywords act as "sources codes" which are used to retrieve the relevant published literature from an infinite database of knowledge. ${ }^{43,44}$ We identified the most commonly used keywords relevant to the dental caries to enable and facilitate study of dental caries through different search engines. "Fluoride" was identified as the fourth most commonly used keyword by authors after "Streptococcus mutans." The presence of increased levels of Streptococcus mutans in saliva has been correlated with an increased prevalence of caries in numerous studies. ${ }^{45,46}$ Therapeutic effect of fluoride on reducing the prevalence of dental caries has been widely reported. ${ }^{47,48}$ Nevertheless, limitation of dosage according to the recommendations plays a key role to avoid further complications. ${ }^{49}$ 
vosviewer

Fig. 4 Keyword network analysis.

Although it is questionable to judge the validity of a publication based on citation counts, a strong association between impact on a field and high citation counts has been reported. ${ }^{50}$ Factors which influence the counts of citation of a publication include recitation of highly cited article, author reputation in a specific field, and preference of a specific (reputable) journal. The factors described above and others make the number of citations an impartial reflector of the impact and quality of a publication. Modern day platforms such as hashtags, likes, tweets, shares, downloads, and trends depict the influence of electronic media and the impact of a publication on a day-to-day basis which might project a publication as the most cited within the first 3 days of its publication..$^{51}$

The strengths of this study are: an accepted and commonly used technical methodology, high interrater reliability due to simple inclusion and exclusion criteria, and quality control through two independent investigators and discussion to resolve inconsistencies. The limitations of this study include the fact that the WoS "All Databases" did not measure citations for articles published before 1945. Hence, there might be a possibility of omission of important publications before this date. Second, a publication can have many coauthors from different regions of the world, but the address and affiliation of the corresponding author could only be recognized and has been documented which could have resulted in the possible contributions from other countries and institutions of the world being missed. Third, only the articles published in indexed journals were included: however, it appears unlikely that such a publication would have a high citation count.

\section{Conclusion}

This is the first study reporting the list of top 100 "classics" on dental caries. Citation density and citation count did not have a significant association with the publication year. This study despite its inherent limitations attempts to provide a quantitative measure of the impact that a publication imparts on its relevant specialty/field.

\section{Funding}

The first author is grateful to the university for providing financial assistance under fellowship scheme for 2 years of his candidature. All authors acknowledge the sponsorship of article publication charges from the Universiti Sains Malaysia RU grant no. 1001/PPSG/812079.

\section{Conflict of Interest}

None declared.

\section{References}

1 Pitts NB, Zero DT, Marsh PD, et al. Dental caries. Nat Rev Dis Primers 2017;3(1):17030

2 Hernández-Sierra JF, Ruiz F, Pena DCC, et al. The antimicrobial sensitivity of Streptococcus mutans to nanoparticles of silver, zinc oxide, and gold. Nanomedicine (Lond) 2008;4(3):237-240

3 World Health Organization. World Oral Health Report. Published 2018. Available at: https://www.who.int/news-room/ fact-sheets/detail/oral-health. Accessed February 11, 2020

4 Andrian S, Stoleriu S, Tărăboanță I, Gamen AC, Dimbu E, Negraia D. Remineralization of incipient enamel lesions using non-fluoride agents. A review. IJMD 2018;8(1):41-49

5 Ladewig NM, Camargo LB, Tedesco TK, et al. Management of dental caries among children: a look at the cost-effectiveness. Expert Rev Pharmacoecon Outcomes Res 2018;18(2):127-134 
6 Pedersen AM, Bardow A, Jensen SB, Nauntofte B. Saliva and gastrointestinal functions of taste, mastication, swallowing and digestion. Oral Dis 2002;8(3):117-129

7 Psoter WJ, Reid BC, Katz RV. Malnutrition and dental caries: a review of the literature. Caries Res 2005;39(6):441-447

8 Steeg PS. Perspectives on classic article: metastasis suppressor genes. J Natl Cancer Inst 2004;96(6):E4-E4

9 Park KM, Park BS, Park S, Yoon DY, Bae JS. Top-100 cited articles on headache disorders: a bibliometric analysis. Clin Neurol Neurosurg 2017;157:40-45

10 Aslam-Pervez N, Lubek JE. Most cited publications in oral and maxillofacial surgery: a bibliometric analysis. Oral Maxillofac Surg 2018;22(1):25-37

11 Ullah R, Zafar MS, Riaz I, Hasan SJ. Top cited publications on fluoride in relation to oral health: a bibliometric analysis. Fluoride 2019;52(3):426-446

12 Corbella S, Francetti L, Taschieri S, Weinstein R, Del Fabbro M. Analysis of the 100 most-cited articles in periodontology. J Investig Clin Dent 2017;8(3):e12222

13 Fardi A, Kodonas K, Gogos C, Economides N. Top-cited articles in endodontic journals. J Endod 2011;37(9):1183-1190

14 Fardi A, Kodonas K, Lillis T, Veis A. Top-cited articles in implant dentistry. Int J Oral Maxillofac Implants 2017;32(3):555-564

15 Feijoo JF, Limeres J, Fernández-Varela M, Ramos I, Diz P. The 100 most cited articles in dentistry. Clin Oral Investig 2014;18(3):699-706

16 Van Eck NJ, Waltman L. Bibliometric mapping of the computational intelligence field. Int $\mathrm{J}$ Uncertain Fuzz 2007;15(5):625-645

17 Jafarzadeh H, Sarraf Shirazi A, Andersson L. The most-cited articles in dental, oral, and maxillofacial traumatology during 64 years. Dent Traumatol 2015;31(5):350-360

18 Ahmad P, Alam MK, Jakubovics NS, Schwendicke F, Asif JA. 100 years of the Journal of Dental Research: a bibliometric analysis. J Dent Res 2019;98(13):1425-1436

19 Pena-Cristóbal M, Diniz-Freitas M, Monteiro L, Diz Dios P, Warnakulasuriya S. The 100 most cited articles on oral cancer. J Oral Pathol Med 2018;47(4):333-344

20 Tarazona B, Lucas-Dominguez R, Paredes-Gallardo V, Alonso-Arroyo A, Vidal-Infer A. The 100 most-cited articles in orthodontics: a bibliometric study. Angle Orthod 2018;88(6):785-796

21 Shamseer L, Moher D, Clarke M, et al; PRISMA-P Group. Preferred reporting items for systematic review and meta-analysis protocols (PRISMA-P) 2015: elaboration and explanation. BMJ 2015;350:g7647

22 Loesche WJ. Role of Streptococcus mutans in human dental decay. Microbiol Rev 1986;50(4):353-380

23 Ajdić D, McShan WM, McLaughlin RE, et al. Genome sequence of Streptococcus mutans UA159, a cariogenic dental pathogen. Proc Natl Acad Sci U S A 2002;99(22):14434-14439

24 Ismail AI, Sohn W, Tellez M, et al. The International Caries Detection and Assessment System (ICDAS): an integrated system for measuring dental caries. Community Dent Oral Epidemiol 2007;35(3):170-178

25 Kassebaum NJ, Bernabé E, Dahiya M, Bhandari B, Murray CJ, Marcenes W. Global burden of untreated caries: a systematic review and metaregression. J Dent Res 2015;94(5):650-658

26 Gustafsson BE, Quensel CE, Lanke LS, et al. The Vipeholm dental caries study; the effect of different levels of carbohydrate intake on caries activity in 436 individuals observed for five years. Acta Odontol Scand 1954;11(3-4):232-264

27 Andersen J, Belmont J, Cho CT. Journal impact factor in the era of expanding literature. J Microbiol Immunol Infect 2006;39(6):436-443

28 Ahmad P, Dummer PMH, Noorani TY, Asif JA. The top 50 most-cited articles published in the International Endodontic Journal. Int Endod J 2019;52(6):803-818
29 Ugolini D, Neri M, Cesario A, et al. Scientific production in cancer rehabilitation grows higher: a bibliometric analysis. Support Care Cancer 2012;20(8):1629-1638

30 Baltussen A, Kindler CH. Citation classics in critical care medicine. Intensive Care Med 2004;30(5):902-910

31 Baltussen A, Kindler CH. Citation classics in anesthetic journals. Anesth Analg 2004;98(2):443-451

32 Fenton JE, Roy D, Hughes JP, Jones AS. A century of citation classics in otolaryngology-head and neck Surgery journals. J Laryngol Otol 2002;116(7):494-498

33 Paladugu R, Schein M, Gardezi S, Wise L. One hundred citation classics in general surgical journals. World J Surg 2002;26(9):1099-1105

34 Uthman OA, Okwundu CI, Wiysonge CS, Young T, Clarke A. Citation classics in systematic reviews and meta-analyses: who wrote the top 100 most cited articles? PLoS One 2013;8(10):e78517

35 Brandt JS, Downing AC, Howard DL, Kofinas JD, Chasen ST. Citation classics in obstetrics and gynecology: the 100 most frequently cited journal articles in the last 50 years. Am J Obstet Gynecol 2010;203(4):355.e1-355.e7

36 Lefaivre KA, Guy P, O’Brien PJ, Blachut PA, Shadgan B, Broekhuyse HM. Leading 20 at 20: top cited articles and authors in the Journal of Orthopaedic Trauma, 1987-2007. J Orthop Trauma 2010;24(1):53-58

37 Loonen MP, Hage JJ, Kon M. Plastic Surgery Classics: characteristics of 50 top-cited articles in four Plastic Surgery Journals since 1946. Plast Reconstr Surg 2008;121(5):320e-327e

38 Shadgan B, Roig M, Hajghanbari B, Reid WD. Top-cited articles in rehabilitation. Arch Phys Med Rehabil 2010;91(5):806-815

39 Campbell FM. National bias: a comparison of citation practices by health professionals. Bull Med Libr Assoc 1990;78(4):376-382

40 Daly J, Willis K, Small R, et al. A hierarchy of evidence for assessing qualitative health research. J Clin Epidemiol 2007;60(1):43-49

41 Kulkarni AV, Aziz B, Shams I, Busse JW. Comparisons of citations in Web of Science, Scopus, and Google Scholar for articles published in general medical journals. JAMA 2009;302(10):1092-1096

42 Brookes BC. Bradford's law and the bibliography of science. Nature 1969;224(5223):953-956

43 Natarajan K, Stein D, Jain S, Elhadad N. An analysis of clinical queries in an electronic health record search utility. Int J Med Inform 2010;79(7):515-522

44 Asghari S, Navimipour NJ. Nature inspired meta-heuristic algorithms for solving the service composition problem in the cloud environments. Int J Commun Syst 2018;31(12):e3708

45 Pannu P, Gambhir R, Sujlana A. Correlation between the salivary Streptococcus mutans levels and dental caries experience in adult population of Chandigarh, India. Eur J Dent 2013;7(2):191-195

46 Kocak MM, Ozcan S, Kocak S, Topuz O, Erten H. Comparison of the efficacy of three different mouthrinse solutions in decreasing the level of streptococcus mutans in saliva. Eur J Dent 2009;3(1):57-61

47 Al-Akwa AA, Al-Maweri SA. Dental caries prevalence and its association with fluoride level in drinking water in Sana'a, Yemen. Eur J Dent 2018;12(1):15-20

48 Zafar MS, Ahmed N. Therapeutic roles of fluoride released from restorative dental materials. Fluoride 2015;48(3):184-194

49 Ullah R, Zafar MS, Shahani N. Potential fluoride toxicity from oral medicaments: a review. Iran J Basic Med Sci 2017;20(8):841-848

50 Ioannidis JP, Boyack KW, Small H, Sorensen AA, Klavans R. Bibliometrics: is your most cited work your best? Nature 2014;514(7524):561-562

51 Eysenbach G. Can tweets predict citations? Metrics of social impact based on Twitter and correlation with traditional metrics of scientific impact. J Med Internet Res 2011;13(4):e123 